University of New Hampshire

University of New Hampshire Scholars' Repository

Space Science Center

Institute for the Study of Earth, Oceans, and

Space (EOS)

2000

\title{
Milagro: A TeV gamma-ray monitor of the Northern Hemisphere Sky
}

\author{
B L. Dingus \\ University of Utah \\ R Atkins \\ University of Utah \\ W Benbow \\ University of California - Santa Cruz \\ D Berley \\ University of Maryland - College Park \\ M L. Chen \\ University of Maryland - College Park \\ Follow this and additional works at: https://scholars.unh.edu/ssc \\ Part of the Astrophysics and Astronomy Commons \\ See next page for additional authors
}

\section{Recommended Citation}

Milagro: A TeV gamma-ray monitor of the Northern Hemisphere Sky Dingus, B. L. and Atkins, R. and Benbow, W. and Berley, D. and Chen, M. L. and Coyne, D. G. and Dorfan, D. E. and Ellsworth, R. W. and Evans, D. and Falcone, A. and Fleysher, L. and Fleysher, R. and Gisler, G. and Goodman, J. A. and Haines, T. J. and Hoffman, C. M. and Hugenberger, S. and Kelley, L. A. and Leonor, I. and McConnell, M. and McCullough, J. F. and McEnery, J. E. and Miller, R. S. and Mincer, A. I. and Morales, M. F. and Nemethy, P. and Ryan, J. M. and Shen, B. and Shoup, A. and Sinnis, C. and Smith, A. J. and Sullivan, G. W. and Tumer, T. and Wang, K. and Wascko, M. O. and Westerhoff, S. and Williams, D. A. and Yang, T. and Yodh, G. B., AIP Conference Proceedings, 510, 642-645 (2000), DOI:http://dx.doi.org/10.1063/1.1303280

This Conference Proceeding is brought to you for free and open access by the Institute for the Study of Earth, Oceans, and Space (EOS) at University of New Hampshire Scholars' Repository. It has been accepted for inclusion in Space Science Center by an authorized administrator of University of New Hampshire Scholars' Repository. For more information, please contact Scholarly.Communication@unh.edu. 


\section{Authors}

B L. Dingus, R Atkins, W Benbow, D Berley, M L. Chen, D G. Coyne, D E. Dorfan, R W. Ellsworth, D Evans, A Falcone, L Fleysher, R Fleysher, G Gisler, J A. Goodman, T J. Haines, C M. Hoffman, S Hugenberger, L A. Kelley, I Leonor, Mark L. McConnell, J F. McCullough, J E. McEnery, R S. Miller, A I. Mincer, M F. Morales, P Nemethy, James M. Ryan, B Shen, A Shoup, C Sinnis, A J. Smith, G W. Sullivan, O T. Tumer, K Wang, M O. Wascko, S Westerhoff, D A. Williams, T Yang, and G B. Yodh 


\section{AIP $\mid$ Proceedings}

\section{Milagro: A TeV gamma-ray monitor of the Northern Hemisphere Sky}

B. L. Dingus, R. Atkins, W. Benbow, D. Berley, M. L. Chen, D. G. Coyne, D. E. Dorfan, R. W. Ellsworth, D. Evans , A. Falcone, L. Fleysher, R. Fleysher, G. Gisler, J. A. Goodman, T. J. Haines, C. M. Hoffman, S. Hugenberger, L. A. Kelley, I. Leonor, M. McConnell, J. F. McCullough, J. E. McEnery, R. S. Miller, A. I. Mincer, M. F. Morales, P. Nemethy, J. M. Ryan, B. Shen, A. Shoup, C. Sinnis, A. J. Smith, G. W. Sullivan, T. Tumer, K. Wang, M. O. Wascko, S. Westerhoff, D. A. Williams, T. Yang, and G. B. Yodh

Citation: AIP Conference Proceedings 510, 642 (2000); doi: 10.1063/1.1303280

View online: http://dx.doi.org/10.1063/1.1303280

View Table of Contents: http://scitation.aip.org/content/aip/proceeding/aipcp/510?ver=pdfcov Published by the AIP Publishing

Articles you may be interested in

A "Snapshot" survey of the gamma-ray sky at GeV energies

AIP Conf. Proc. 510, 611 (2000); 10.1063/1.1303275

First results of a study of TeV emission from GRBs in Milagrito

AIP Conf. Proc. 510, 543 (2000); 10.1063/1.1303263

A search for gamma-ray burst optical emission with the automated patrol telescope AIP Conf. Proc. 428, 909 (1998); 10.1063/1.55461

Searches for TeV counterparts to classical gamma-ray bursts AIP Conf. Proc. 384, 603 (1996); 10.1063/1.51577

MILAGROA TeV air shower array AIP Conf. Proc. 220, 275 (1991); 10.1063/1.40309 


\title{
Milagro: A TeV Gamma-Ray Monitor of the Northern Hemisphere Sky
}

\author{
B.L. Dingus ${ }^{1}$, R. Atkins ${ }^{1}$, W. Benbow ${ }^{2}$, D. Berley ${ }^{3,10}$, \\ M.L. Chen ${ }^{3,11}$, D.G. Coyne ${ }^{2}$, D.E. Dorfan ${ }^{2}$, R.W. Ellsworth ${ }^{5}$, \\ D. Evans ${ }^{3}$, A. Falcone ${ }^{6}$, L. Fleysher ${ }^{7}$, R. Fleysher ${ }^{7}$, G. Gisler ${ }^{8}$, \\ J.A. Goodman ${ }^{3}$, T.J. Haines ${ }^{8}$, C.M. Hoffman ${ }^{8}$, S. Hugenberger ${ }^{4}$, \\ L.A. Kelley ${ }^{2}$, I. Leonor ${ }^{4}$, M. McConnell ${ }^{6}$, J.F. McCullough ${ }^{2}$, \\ J.E. McEnery ${ }^{1}$, R.S. Miller ${ }^{8,6}$, A.I. Mincer ${ }^{7}$, M.F. Morales ${ }^{2}$, \\ P. Nemethy ${ }^{7}$, J.M. Ryan ${ }^{6}$, B. Shen ${ }^{9}$, A. Shoup ${ }^{4}$, C. Sinnis ${ }^{8}$, \\ A.J. Smith ${ }^{9}$, G.W. Sullivan ${ }^{3}$, T. Tumer ${ }^{9}$, K. Wang ${ }^{9}$, M.O. Wascko ${ }^{9}$,

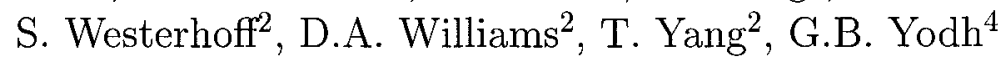 \\ (1) University of Utah, Salt Lake City, UT 84112, USA \\ (2) University of California, Santa Cruz, CA 95064, USA \\ (3) University of Maryland, College Park, MD 20742, USA \\ (4) University of California, Irvine, CA 92697, USA \\ (5) George Mason University, Fairfax, VA 22030, USA \\ (6) University of New Hampshire, Durham, NH03824, USA \\ (7) New York University, New York, NY10003, USA \\ (8) Los Alamos National Laboratory, Los Alamos, NM 87545, USA \\ (9) University of California, Riverside, CA 92521, USA \\ (10) Permanent Address: National Science Foundation, Arlington, VA, 22230, USA \\ (11) Now at Brookhaven National Laboratory, Upton, NY11979, USA

\begin{abstract}
A new type of very high energy (>a few $100 \mathrm{GeV}$ ) gamma-ray observatory, Milagro, has been built with a large field of view of $>1$ steradian and nearly 24 hours/day operation. Milagrito, a prototype for Milagro, was operated from February 1997 to May 1998. During the summer of 1998, Milagrito was dismantled and Milagro was built. Both detectors use a $80 \mathrm{~m} \times 60 \mathrm{~m} \times 8 \mathrm{~m}$ pond of water in which a $3 \mathrm{~m} \mathrm{x}$ $3 \mathrm{~m}$ grid of photomultiplier tubes detects the Cherenkov light produced in the water by the relativistic particles in extensive air showers. Milagrito was smaller and had only one layer of photomultipliers, but allowed the technique to be tested. Milagrito observations of the Moon's shadow and Mrk 501 are consistent with the Monte Carlo prediction of the telescopes parameters, such as effective area and angular resolution. Milagro is larger and consists of two layers of photomultiplier tubes. The bottom layer detects penetrating particles that are used to reject the background of cosmic-ray initiated showers.
\end{abstract}

CP510, The Fifth Compton Symposium, edited by M. L. McConnell and J. M. Ryan (C) 2000 American Institute of Physics 1-56396-932-7/00/\$17.00 


\section{A NEW TYPE OF TEV $\gamma$-RAY OBSERVATORY}

Several active galactic nuclei and supernova remnants have now been observed to emit $\mathrm{TeV}$ gamma-rays. [1], [2], [3] These $\mathrm{TeV}$ observations were made by detecting the atmospheric Cherenkov light produced by the extensive air shower of particles created when a high energy gamma ray interacts in the atmosphere. Due to the limitations of this detection technique, these observatories only operate on clear, moonless nights and must point at the source using a large mirror. Therefore, observations of unpredictable, short duration transients, such as gamma-ray bursts, and all sky surveys are difficult.

A new type of $\mathrm{TeV} \gamma$-ray observatory with a large field of view and continuous operation has recently been built in the Jemez Mountains near Los Alamos, NM $\left(106.7^{\circ} \mathrm{W}, 35.9^{\circ} \mathrm{N}, 2650 \mathrm{~m}\right.$ above sea level). The observatory is called Milagro, and the prototype which operated at the same site from February 1997 to May 1998 is called Milagrito [4].

Both detectors used the large pond of water $80 \mathrm{~m} \times 60 \mathrm{~m} \times 8 \mathrm{~m}$ which can be seen in the photograph of Figure 1. The pond has a light-tight cover. Milagro contains 723 photomultiplier tubes (PMTs) which are placed on a $3 \mathrm{~m} \times 3 \mathrm{~m}$ grid in 2 layers at $1.5 \mathrm{~m}$ and $7 \mathrm{~m}$ below the surface. The prototype Milagrito had only one layer of 228 PMTs on a $3 \mathrm{~m} \times 3 \mathrm{~m}$ grid spread over the smaller area of $30 \mathrm{~m} \mathrm{x}$ $50 \mathrm{~m}$.

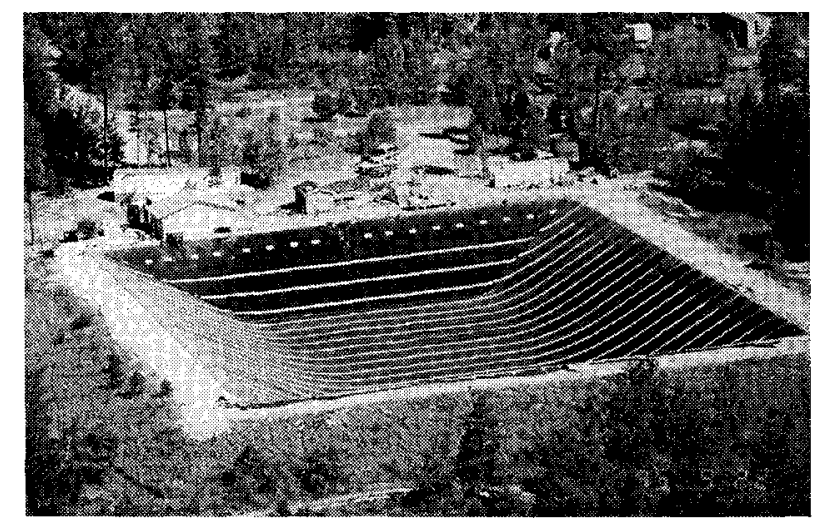

FIGURE 1. Aerial photograph of the $60 \mathrm{~m} \times 80 \mathrm{~m} \times 8 \mathrm{~m}$ pond and cover used by Milagro and Milagrito. The pond as instrumented for Milagro contains 5 million gallons of water.

An extensive air shower is detected when the relativistic particles radiate Cherenkov light in the water causing several tens of PMTs to observe the light within a few $100 \mathrm{nsec}$ of each other. From the relative timing of the photomultiplier tube signals, the direction of the particle or gamma-ray initiating the shower can be determined to $\sim 1$ degree depending on the number of PMTs hit. The field of view is such that $50 \%$ of the showers detected are within 20 degrees of zenith and 
$90 \%$ are within 50 degrees. Almost all of these triggers are due to the background of showers that are initiated by charged cosmic rays. Monte Carlo simulations correctly predict the observed rate and zenith angle distribution of cosmic-ray initiated showers. Simulations of $\gamma$-ray initiated showers show sensitivity to $\gamma$-rays as low as $\sim 100 \mathrm{GeV}$ with the effective area increasing as $\mathrm{E}^{\sim 2}$, where $\mathrm{E}$ is the $\gamma$-ray energy, and flattening near $10 \mathrm{TeV}$.

\section{MILAGRITO OBSERVATIONS}

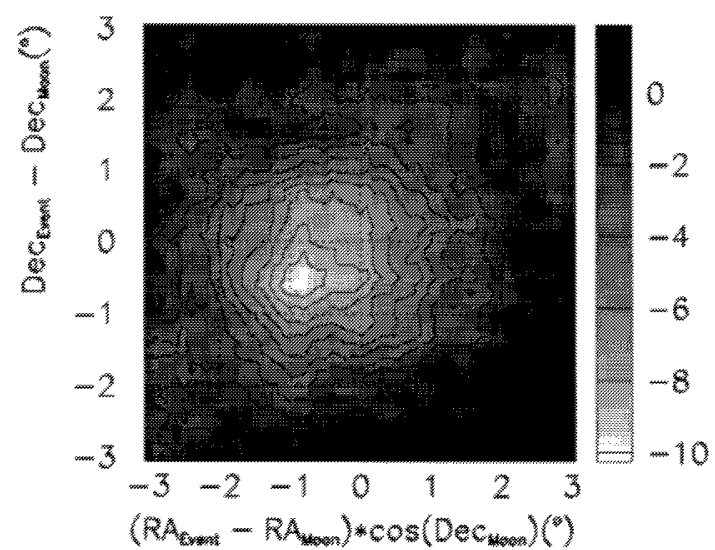

FIGURE 2. The shadow of the Moon due to the blockage of cosmic rays. The scale is in standard deviations of the Gaussian distributed background. The deficit is not located at the direction to the Moon, but is deflected in R.A. because the trajectories of the charged cosmic rays are bent by the Earth's magnetic field. a 3.7 sigma deviation from the cosmic-ray background [6]. The flux and spectrum were well measured by several atmospheric Cherenkov telescopes and the significance of the Milagrito detection agrees with this spectrum folded with the Milagrito effective area. Simulations also indicate that the sensitivity of Milagrito was too low to detect the Crab nebula, the standard candle of $\mathrm{TeV}$ astronomy.

New observations have been performed by Milagrito that atmospheric Cherenkov telescopes have not been able to do because of their low duty cycle (5-10\%) and small field of view (2-4 degrees in radius). Specifically, an all sky survey of the Northern Hemisphere was performed and no sources brighter than 5 times the Crab nebular flux were detected [7]. An all-sky search for 10 second duration transients was also done and none were found [8], which can place limits on the local density of evaporating primordial black holes. However, the most interesting results have 


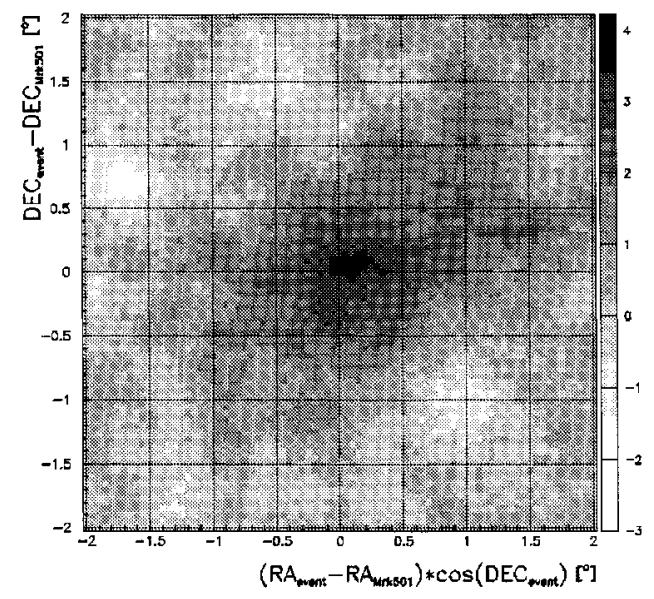

FIGURE 3. The number of showers in the vicinity of Mrk501 plotted in standard deviations of the background. For each position the number of showers is determined for directions within one degree of that position; thus neighboring bins are highly correlated. The position of Mrk 501 is at the center of the plot. The excess at Mrk 501 is $3624 \pm 990$ events, or $3.7 \sigma$.

been from the search for TeV emission correlated with BATSE detected bursts [9] and the detection of a coronal mass ejection from the Sun [10]. These analyses were both reported separately at this conference.

\section{MILAGRO EXPECTATIONS}

Simulations and preliminary data indicate that Milagro will be more than 5 times more sensitive than Milagrito. The improvement comes from a combination of factors - larger area, improved angular resolution, and cosmic-ray background rejection. Milagro will be fully operational Fall 1999 with a data taking rate of 2000 showers per second resulting in more than 100 GBytes of data per day.

\section{REFERENCES}

1. M. Catanese \& T.C. Weekes, Astronomical Society of the Pacific (1999) (in press)

2. C.M. Hoffman, C. Sinnis, P. Fleury, M. Punch Rev. of Mod. Phys. $71(4), 897$ (1999)

3. R. Ong Physics Reports 305, 93 (1998)

4. R. Atkins et al., submitted to Nucl. Inst. and Methods (2000)

5. M. Wascko et al., 26th ICRC Proceedings (Salt Lake City,UT) (1999)

6. R. Atkins et al., Ap. J. Lett. 525, L25 (1999).

7. A.J. Smith et al. AIP Proc. GeV-TeV Gamma-Ray Astrophysics-Toward a Major Atmospheric Cherenkov Telescope VI (Snowbird, UT) (2000)

8. C. Sinnis et al., 26th ICRC Proceedings (Salt Lake City,UT) (1999)

9. R. S. Miller et al., AIP Proc. 5th Compton Symp. (Portsmouth,NH) (2000)

10. A. Falcone et al., AIP Proc. 5th Compton Symp. (Portsmouth,NH) (2000) 